\title{
Does Evidence Permeate All Surgical Areas Equally? Publication Trends in Wound Care Compared to Breast Cancer Care: A Longitudinal Trend Analysis
}

\author{
F. E. Brölmann • M. D. Groenewold • \\ R. Spijker · J. A. van der Hage • D. T. Ubbink • \\ H. Vermeulen
}

Published online: 19 April 2012

(C) The Author(s) 2012. This article is published with open access at Springerlink.com

\begin{abstract}
Background Evidence-based decision making has permeated the daily practice of healthcare professionals. However, in wound care this seems more difficult than in other medical areas, such as breast cancer, which has a similar incidence, variety of etiologies, financial burden, and diversity of treatment options. This incongruence could be due to a lack in quantity and quality of available evidence. We therefore compared worldwide publication
\end{abstract}

This abstract was presented as a poster presentation at the 24th Annual Symposium on Advanced Wound Care/Wound Healing Society Meeting, April 14-17, 2011, Dallas Texas.

Electronic supplementary material The online version of this article (doi:10.1007/s00268-012-1599-8) contains supplementary material, which is available to authorized users.

F. E. Brölmann · M. D. Groenewold · D. T. Ubbink ( $\square)$.

H. Vermeulen

Departments of Quality Assurance \& Process Innovation, Academic Medical Center, Room A3-503, Meibergdreef 9, P.O Box 22700, 1100 DE Amsterdam, The Netherlands

e-mail: d.ubbink@amc.nl

R. Spijker

Central Medical Library, Academic Medical Center,

Amsterdam, The Netherlands

\section{J. A. van der Hage}

The Netherlands Cancer Institute, Amsterdam, The Netherlands

D. T. Ubbink

Department of Surgery, Academic Medical Center, Amsterdam, The Netherlands

H. Vermeulen

Amsterdam School of Health Professions, University of

Amsterdam, Amsterdam, The Netherlands trends to answer whether research in wound care lags behind that in breast cancer.

Methods In order to assess the trends in quantity and methodological quality of publications as to wound care and breast cancer treatments, we examined relevant publications over the last five decades. Publications in MEDLINE were classified into seven study design categories: (1) guidelines, (2) systematic reviews (SR), (3) randomized (RCT), and controlled clinical trials (CCT), (4) cohort studies, (5) case-control studies, (6) case series and case reports, and (7) other publications.

Results We found a 30-fold rise in publications on wound care, versus a 70-fold increase in those on breast cancer. High-quality study designs like SR, RCT, or CCT were less frequent in wound care (difference 1.9, $95 \% \mathrm{CI}$ $1.8-2.0 \%$ ) as were guidelines; 76 on wound care versus 231 for breast cancer.

Conclusions Publications on wound care fall behind in quantity and quality as compared to breast cancer. Nevertheless, SR, RCT, and CCT in wound care are becoming more numerous. These high-quality study designs could motivate clinicians to make evidence-based decisions and researchers to perform proper research in wound care.

\section{Introduction}

Every day, surgeons are charged with solving decisional dilemmas while taking care of their patients. Ideally, such choices are based on best available evidence, clinical expertise, and patient preferences. This evidence-based decision making has gradually permeated the daily practice of modern healthcare professionals [1-3] and is endorsed by the U.S. National Institute of Medicine [4]. It is safe to say that nowadays no surgical area is exempt from the 
obligation to generate and use convincing evidence in the practice high-quality patient care. However, the principle of evidence-based practice has not been implemented equally among all surgical areas [5]. For example, in wound care, evidence-based decision making seems to flourish less than in many other medical areas [6]. The reason for this is unclear, particularly considering the financial impact, prevalence, and effect on quality of life that make wound care a serious health care burden that needs to be relieved by proper evidence 7-11]

A representative illustration of the situation in another surgical area is found in breast cancer. This is a disorder in which huge amounts of money have been invested for research purposes. Although this disorder is obviously different from wounds, it has remarkable similarities in terms of being a surgical disorder characterized by a large diversity of etiologies, treatment options, and outcomes measured [12]. In addition, the lifetime risks of acquiring breast cancer or a (chronic) wound are similar; roughly one of out of every ten subjects [7, 13-18]. Hence, one might think these two disorders deserve equal research efforts and similarly sized bodies of knowledge to enable evidencebased decision making.

Any discrepancy in evidence-based decision making between the areas of breast cancer and wound care could be due to a difference in the amount of convincing evidence available. Such evidence is preferably derived from systematic reviews (SR), randomized (RCT), or controlled clinical trials (CCT) [2]. However, particularly in the realm of wound care, opinion-based articles conclude that the mainstay of evidence seems to consist of noncomparative research designs, which are much more sensitive to bias $[6,12,19,20]$. This is articulated by frequent appeals in the conclusion of Cochrane systematic reviews: "evidence is weak, so further research is required to validate these findings" [21-25].

We hypothesize that a lack of convincing evidence in wound care forms a barrier for surgeons to practice evidence-based healthcare. Because the quantity and quality of evidence play a crucial role in decision making, it is interesting to know whether and why empirical evidence features more largely in some medical areas than in others. For this reason, we analyzed and compared the worldwide trends as to the quantity and quality of publications regarding wound care and breast cancer, to answer the following question: Is wound care research behind the times in terms of good quality publication output as compared to breast cancer? The answer to our research question could provide surgeons with information about whether high-quality evidence is available for wounds to promote evidence-based practice in wound care to the same degree that applies in breast cancer. This will also help surgeons with clinical and economical decision making to ensure optimum quality of care.

\section{Methods}

We identified all relevant scientific publications over the last 5 decades concerning wound and breast cancer treatments. We did not exclude publication types like letters, editorials, or comments because publication types incorrectly tagged could be missed using search filters [26] Search strategies were designed in cooperation with a medical information specialist. We searched MEDLINE from 1961 to 2010 by means of two interfaces: OVID for a wide-ranging search of all publication types, followed by PubMed to find particular guidelines. The general search strategies from the Cochrane Wounds Group and the Cochrane Breast Cancer Group were used (see Electronic Supplementary Material). To distinguish the various study designs, these searches were combined with filters available from the BMJ (British Medical Journal) Evidence Centre, the Cochrane Collaboration and Scottish Intercollegiate Guidelines Network (SIGN) (see Electronic Supplementary Material). We did not apply any search limitations such as publication year, type of article, or language.

Subsequently, the selected publications were classified into one of seven study design categories: (1) guidelines, (2) SR, (3) RCT and CCT, (4) cohort studies, (5) casecontrol studies, (6) case series and case reports, and (7) other publications. Realizing that the available filters for specific study designs are not perfect [26, 27], we validated our search strategy by means of spot-checks of the publications found in both disorders. For this purpose, we randomly chose 100 publications from each study design and in three different 5-year periods to validate the search filter. Titles and abstracts were screened independently by two researchers as to which study design was used and whether this matched the filters used. All search strategies were adapted until the highest number of correct study designs was found with the lowest number of erroneous ones. Adaptations were made by excluding MESH terms like "*peptic ulcer/" "*colitis, ulcerative/" "*eye infections", or by adding terms like "wound\$.ti" and "traumatic wound\$.ti”.

Finally, PubMed was independently searched by two researchers to find guidelines. These were checked for relevance; i.e., they should address screening, prevention, etiology, pathology, diagnosis, or treatment.

\section{Data analysis}

We calculated how many of the publications found belonged to our predefined publication type categories. The absolute and relative-i.e., in relation to the total in its category—numbers of publications per five years were 
recorded and plotted as frequency histograms against their publication date. Differences in percentages were calculated including their $95 \%$ confidence intervals (CI).

\section{Results}

Quantity and quality of publications

Over the last 5 decades we found a total of 145,114 publications on wound care and 217,484 on breast cancer treatment. For wound and breast cancer treatment alike, the majority of publications were classified as "other publications" (65.6 vs. $72.5 \%$, respectively), as detailed in the paragraph below.

Differences in quality are illustrated in Fig. 1, which gives an overview of the different study designs (categories 2-6). Studies on wound care were significantly more observational than those on breast cancer (31.2 vs. $22.2 \%$, respectively; difference 9.0, $95 \%$ CI 8.7-9.3). In addition, the proportion of case series and case reports was significantly higher in wound care $(20.5 \%)$ than in breast cancer publications (10.2\%; difference 10.3, $95 \%$ CI 10.0-10.5). Only a very small percentage of the articles (wound care $3.1 \%$; breast cancer $5.3 \%$ ) could be classified as SR, $\mathrm{RCT}$, or CCT, but significantly more on breast cancer (difference 2.16, $95 \%$ CI 2.03-2.29). Thus, over twice as many RCT and CCT were available on breast cancer treatment $(10,186)$ as on wound care $(4,061)$.

Verification of study categorization: other publications

By means of spot-checks, 100 randomly selected publications in three five-year periods, 1981-1985, 1991-1995, and 2001-2005, were re-categorized by hand, to verify the study type as indicated by the search filter and to check the types of publications grouped in the relatively large

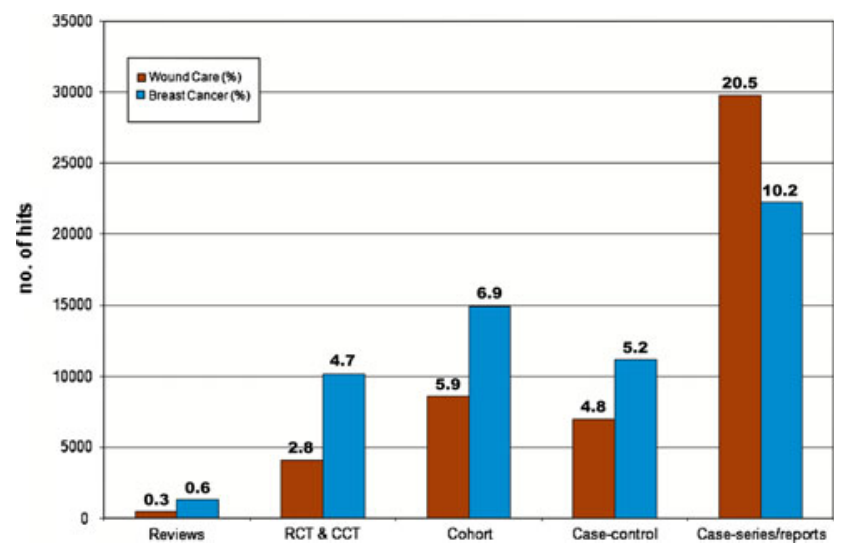

Fig. 1 Total numbers of studies found in wound care and breast cancer for each study design (2-6) during the last 5 decades
Table 1 Result of the spot-checks of category: other publication types

\begin{tabular}{|c|c|c|}
\hline Other publications & $\begin{array}{l}\text { Wound care } \\
\text { records }(\%)\end{array}$ & $\begin{array}{l}\text { Breast cancer } \\
\text { records }(\%)\end{array}$ \\
\hline \multicolumn{3}{|c|}{ Publications incorrectly categorized as "other publications" } \\
\hline Clinical trial & $5(1.6)$ & $3(1.0)$ \\
\hline Observational studies & $62(20.7)$ & $74(24.7)$ \\
\hline Subtotal & $65(21.7)$ & $77(25.7)$ \\
\hline \multicolumn{3}{|c|}{ Publications correctly categorized as "other publications } \\
\hline (Narrative) review & $55(18.3 \%)$ & $70(23.3)$ \\
\hline Pilot evaluation & & $1(0.3)$ \\
\hline Laboratory studies (in vitro) & $60(20.0 \%)$ & $46(15.3)$ \\
\hline Animal studies or plant studies & $19(6.3 \%)$ & $5(1.6)$ \\
\hline Letter, comment, or editorial & $17(5.7 \%)$ & $17(5.7)$ \\
\hline $\begin{array}{l}\text { Unknown (e.g., insufficient } \\
\text { information available) }\end{array}$ & $80(26.7 \%)$ & $83(27.7)$ \\
\hline Economic evaluation & $4(1.3 \%)$ & \\
\hline Subtotal & $235(78.3)$ & $223(74.3)$ \\
\hline Total & $300(100)$ & $300(100)$ \\
\hline
\end{tabular}

category of "other publications." Over $90 \%$ of the category 2 through 6 study types was found to be correctly classified by the search filters. About three quarters of the spot-check publications were confirmed as "other publications" (Table 1). The remainder, $21.7 \%$ in wounds and $25.7 \%$ in breast cancer, were re-categorized as clinical trial or observational study.

Publication trends in time

Figures 2 and 3 show the number of publications between 1961 and October 2010 in 5-year intervals for wound care and breast cancer. During the past 50 years, breast cancer publications showed a higher number and a quicker growth than wound care publications. In both disorders, the numbers of publications increased substantially. However, for wound care this was an approximately 30 -fold increase, whereas for breast cancer it was a 70 -fold increase. This trend was more pronounced for the number of trials published, i.e., 800-fold for wound care and 1,700-fold for breast cancer.

\section{Guidelines}

Of the 211 guidelines found for wound care, only 76 (36\%) guidelines were indeed relevant to wound care. The other guidelines contained a diversity of other medical specialties not related to wounds. In contrast, for breast cancer, 231 (90\%) of the guidelines found were relevant to breast cancer. Figure 4 shows that the number of wound care guidelines increased 5.4 times over the last 5 decades, 


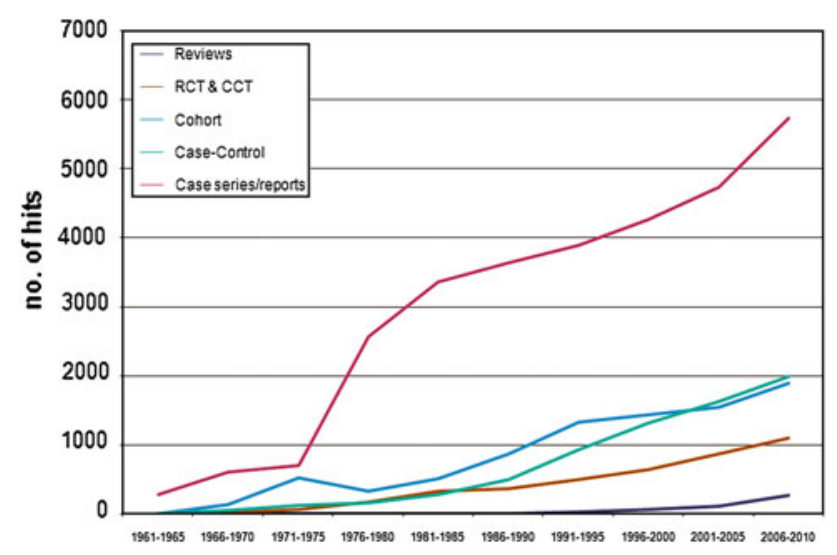

Fig. 2 Wound care publication trends by study design

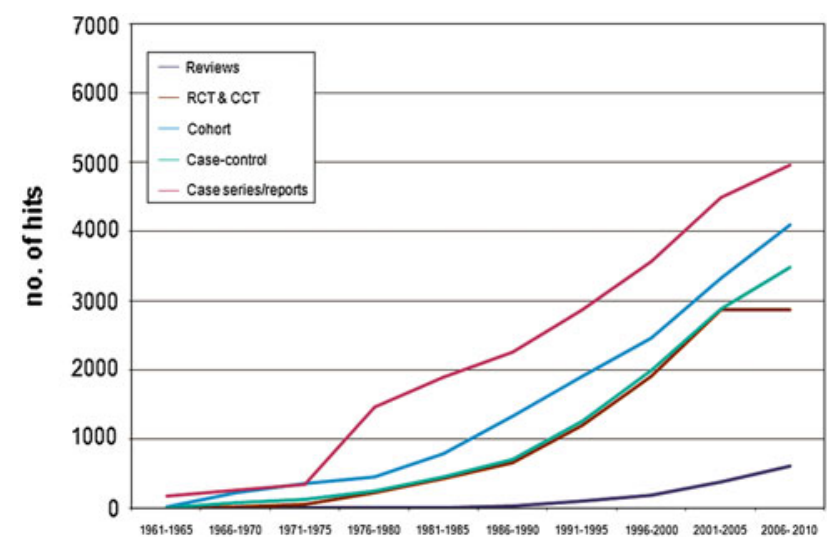

Fig. 3 Breast cancer publication trends by study design

while breast cancer guidelines showed a 15.4-fold increase over the same period of time. Table 2 shows that guidelines for wound care applied mostly to chronic wounds (68\%), rather than acute wounds ( $2 \%)$, prevention, diagnosis, or pathology. Table 3 shows a wide variety in terms of screening, diagnostic, and treatment guidelines (73\%), whereas mammography (4\%) and pathology (4\%) guidelines were less published for breast cancer.

\section{Discussion}

The results of our study confirm a rising number of publications for both wound care and breast cancer, which is no different from other areas in medicine. However, the quantity of publications on breast cancer is larger and has a more exponential character in time as compared to wound care. Also, the quality of studies in terms of robust study designs differs in favor of breast cancer. Significantly more clinical trials and fewer case series or case reports have been reported on breast cancer than on wound care.

We are convinced that these findings present a message that is valuable for surgeons. Although there is an inequality in robust knowledge on wound care compared to

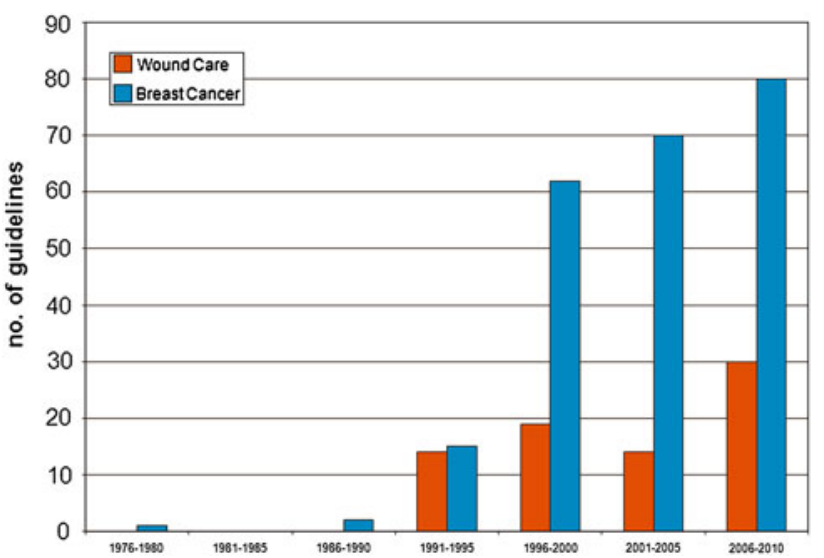

Fig. 4 Guideline trends regarding breast cancer and wound care

other areas, sound evidence is available and should be taken into account by surgeons in their decision making. Wound care and wound healing are of great value to all surgical patients, despite the tendency among some surgeons to consider wounds as a mere tailpiece of surgical procedures. This study should be reason to increase awareness among surgeons of available evidence for wounds.

This study is unique in its kind, as it compares trends in quality and quantity of publication output within these two medical areas. Although no classic examples for this kind of bibliometric research are available to mirror our design and outcomes, we assume our results are likely to be valid. This assumption is based on our use of the generally accepted and sensitive search strategies from the Cochrane Wounds Group and the Cochrane Breast Cancer Group, the spotchecks, and the expertise of our medical information specialist. Furthermore, the spot-checks confirmed the reliability of the different filters used to categorize the studies with exception of the remaining group: other study designs.

Some limitations of our analysis need to be mentioned. First, the searches undertaken as part of this study were performed using the MEDLINE database, which is limited to indexed journals. Wound care research is, probably in contrast to breast cancer research, also distributed through non-indexed journals, which could provide an additional number of case series and case report studies that were not captured in this study. Consequently, our search could have underestimated the proportion of case series and case reports, as well as the total number of wound care publications. When comparing the available high-level evidence in terms of systematic reviews, RCT and CCT, such studies are likely to be published on both disorders alike, possibly fostered by positive publication bias. Adding the attractiveness of breast cancer as a research and societal topic, and the proper scientific evaluation that pharmaceutical treatments for breast cancer require before marketing, it is possible that this kind of research receives more funding 


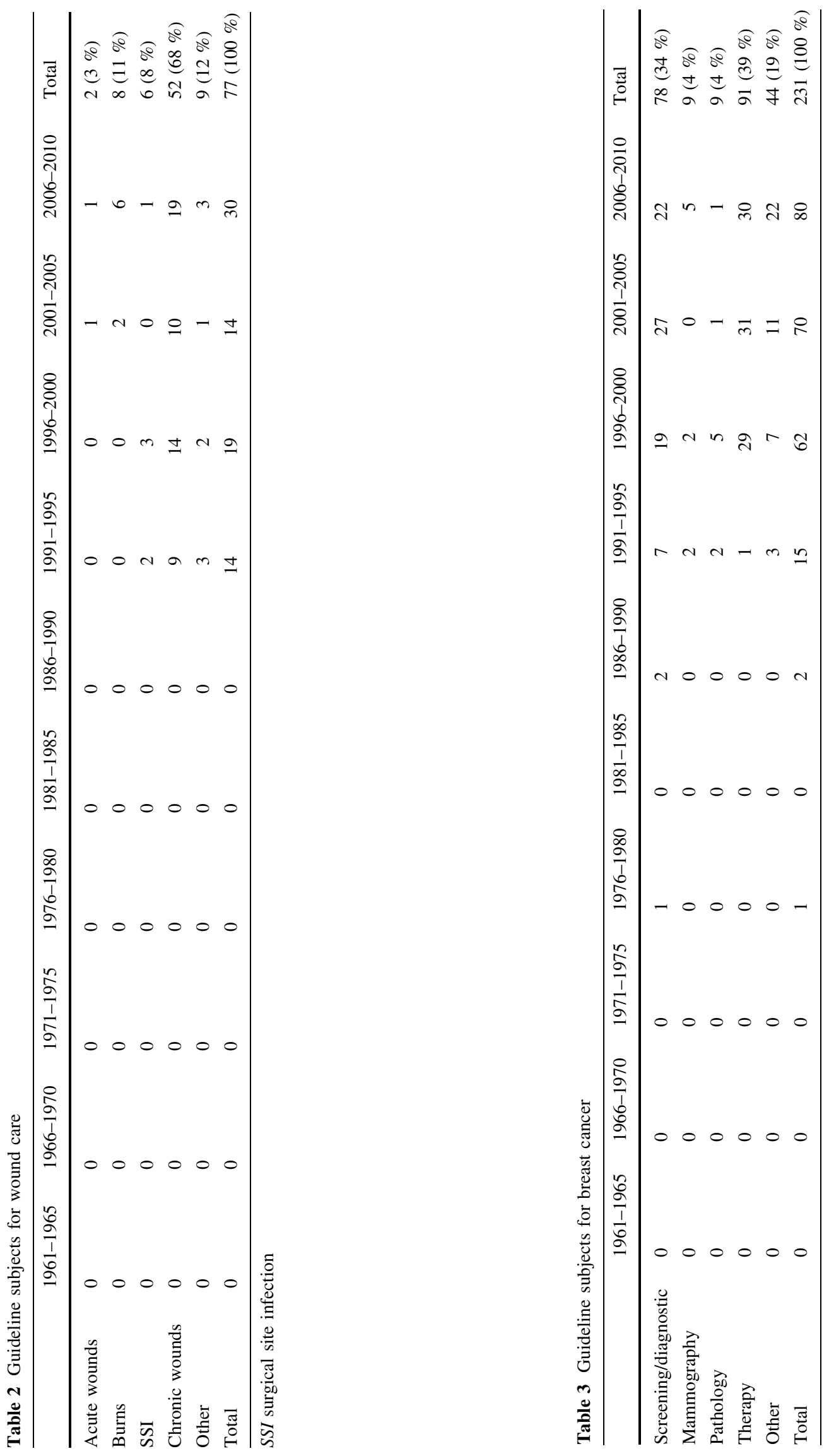


and attention than does wound care and is therefore easier to publish. This study clearly shows a difference in publication output between the disorders for which funding, publication bias, and demand are all plausible causes of these differences.

Second, we limited our analysis to the last five decades. However, the numbers of publications found before 1960 were negligible and unlikely to influence the results of the observed publication trends. Furthermore, our aim was to study overall publication trends, rather than to give a complete historical overview of publications.

Third, it is important to consider the advantages and limitations of a broad search strategy. Its main advantage is a high sensitivity. As a consequence, however, more hits irrelevant to our medical area appeared in such a search strategy, which may have caused an overestimation of the quantity of publications in both areas. We assumed that the number of irrelevant hits would be equally high in both groups and would therefore not interfere with our conclusions. A further limitation of this search strategy might have been the different search strategies used for each medical area. On the other hand, two researchers (M.G. and F.B.) performed the search independently, and their results were similar.

Fourth, the idea of comparing breast cancer to wound care can be questioned. This comparison might seem farfetched, as breast cancer is a malignant, potentially lifethreatening disease while suffering from a wound is not. However, both are very similar in terms of their widespread occurrence, disease burden, and variation in etiology, treatment options, outcome measures, and patients affected. This should be reason for a similar urgency to generate strong evidence regarding their treatments.

Finally, using the recently developed filters to retrieve guidelines in PubMed, we often found duplicate guidelines regarding the same topic or articles that did not include a guideline at all. Even though the same filter was used, this problem appeared larger in wound care. The wound care guidelines reported in this article could therefore be an underestimation of the problems in wound care research and should be further explored to produce new research questions relevant to patients and clinicians.

Although the field of wound care appears somewhat smaller and publications do fall behind in quantity and quality, our analysis shows that systematic reviews-RCT and CCT-in wound care are being performed and are even on the rise in the last decades. This knowledge helps in building arguments against those who claim it is hard to design, conduct, or apply sound research in wound care [28]. The small number of (evidence-based) guidelines for wound care, especially for acute wounds, revealed a niche that has to be addressed in the near future to help clinicians in evidence-based decision making and to facilitate evidence-based medicine in the wound care area.
Acknowledgments We thank David Mulrew for his writing assistance, and the central medical library of the AMC for the literature search.

Open Access This article is distributed under the terms of the Creative Commons Attribution License which permits any use, distribution, and reproduction in any medium, provided the original author(s) and the source are credited.

\section{References}

1. Dickersin K, Straus SE, Bero LA (2007) Evidence based medicine: increasing, not dictating, choice. BMJ 334(Suppl 1):s10

2. Evidence-based Medicine Working Group (1992) Evidencebased medicine: a new approach to teaching the practice of medicine. JAMA 268:2420-2425

3. Montori VM, Guyatt GH (2008) Progress in evidence-based medicine. JAMA 300:1814-1816

4. Committee on Quality of Health Care in America IoM (2001) Crossing the quality chasm: a new healthcare system for the 21st century. Available from: http://www.nap.edu/catalog.php? record_id=10027 Accessed 2-1-2012

5. Knops AM, Vermeulen H, Legemate DA et al (2009) Attitudes, awareness, and barriers regarding evidence-based surgery among surgeons and surgical nurses. World J Surg 33:1348-1355. doi: 10.1007/s00268-009-0020-8

6. Dumville JC, Petherick ES, Cullum N (2008) When will I see you again? The fate of research findings from international wound care conferences. Int Wound J 5:26-33

7. Srinivasaiah N, Dugdall H, Barrett S et al (2007) A point prevalence survey of wounds in north-east England. J Wound Care 16:413-419

8. Mahe E, Langlois G, Baron G et al (2006) Results of a comprehensive hospital-based wound survey. J Wound Care 15: 381-384

9. Vowden K, Vowden P, Posnett J (2009) The resource costs of wound care in Bradford and Airedale primary care trust in the UK. J Wound Care 18(93-98): 100

10. Posnett J, Gottrup F, Lundgren H et al (2009) The resource impact of wounds on health-care providers in Europe. J Wound Care 18:154-161

11. Singer AJ, Dagum AB (2008) Current management of acute cutaneous wounds. N Engl J Med 359:1037-1046

12. Vermeulen H, Ubbink DT, de Zwart F et al (2007) Preferences of patients, doctors, and nurses regarding wound dressing characteristics: a conjoint analysis. Wound Repair Regen 15:302-307

13. National Cancer Institute U.S. (2011) Available from: http://seer. cancer.gov/statfacts/html/breast.html\#risk Accessed 27-4-2011

14. Integraal kankercentrum Zuid IKCnet (2011) Available from: http://www.ikcnet.nl/page.php?id=114 Accessed 27-4-2011

15. Gjorgov AN (1993) Emerging worldwide trends of breast cancer incidence in the 1970s and 1980s: data from 23 cancer registration centres. Eur J Cancer Prev 2:423-440

16. Pieper B, Templin TN, Dobal M et al (1999) Wound prevalence, types, and treatments in home care. Adv Wound Care 12:117-126

17. Posnett J, Franks PJ (2008) The burden of chronic wounds in the UK. Nurs Times 104:44-45

18. Bours GJ, Halfens RJ, Lubbers M et al (1999) The development of a national registration form to measure the prevalence of pressure ulcers in the Netherlands. Ostomy Wound Manage 45(28-38):40

19. Gottrup F (2006) Evidence is a challenge in wound management. Int J Low Extrem Wounds 5:74-75 
20. Leaper D (2009) Evidence-based wound care in the UK. Int Wound J 6:89-91

21. Dryburgh N, Smith F, Donaldson J et al. (2008) Debridement for surgical wounds. Cochrane Database Syst Rev (3)CD006214

22. Jull AB, Rodgers A, Walker N (2008) Honey as a topical treatment for wounds. Cochrane Database Syst Rev (4)CD005083

23. Lethaby A, Temple J, Santy J (2008) Pin site care for preventing infections associated with external bone fixators and pins. Cochrane Database Syst Rev (4)CD004551

24. Vermeulen H, Ubbink D, Goossens A et al. (2004) Dressings and topical agents for surgical wounds healing by secondary intention. Cochrane Database Syst Rev (2)CD003554
25. Wilkinson EA, Hawke CI (2000) Oral zinc for arterial and venous leg ulcers. Cochrane Database Syst Rev (2)CD001273

26. Jenkins M (2004) Evaluation of methodological search filters-a review. Health Info Libr J 21:148-163

27. Sampson M, Zhang L, Morrison A et al (2006) An alternative to the hand searching gold standard: validating methodological search filters using relative recall. BMC Med Res Methodol 6:33

28. Gottrup F, Apelqvist J (2010) The challenge of using randomized trials in wound healing. Br J Surg 97:303-304 